\title{
ESTUDIO DE FENÓMENOS CONSONÁNTICOS DE LA LENGUA ÍBERA*
}

\author{
STUDY OF CONSONANTAL PHENOMENA \\ OF THE ANCIENT IBERIAN LANGUAGE
}

\author{
Jesús RodRíguez Ramos \\ jrr_ib@hotmail.com \\ ORCID: 0000-0001-8772-8149
}

DOI: $10.1387 /$ veleia. 17573

\begin{abstract}
Resumen: El objetivo de este artículo es el estudio de algunos fenómenos del consonantismo de la lengua íbera. El principal punto de partida para la selección de los casos de estudio lo constituyen principalmente las alternancias gráficas en la escritura de determinadas palabras y segmentos, así como la presencia de combinaciones de letras/fonemas excepcionales.

Palabras clave: lengua íbera, lingüística íbera, inscripciones íberas, cambio fonético en la lengua íbera

Abstract: This paper's aim is the phonetical study of some consonantal phenomena of the Ancient Iberian language. The main criteria for the selection of the cases to study are the graphical variations of the writing of some Iberian words and segments, and the presence of unusual combinations of letter/phonemes (such as groups of three vowels).

Keywords: ancient Iberian language, ancient Iberian linguistics, ancient Iberian inscriptions, phonetic change in ancient Iberian language.
\end{abstract}

Recibido: 30/8/2016

Informado: 22/3/2017

Definitivo: $27 / 7 / 2017$

\footnotetext{
* En el texto final hay que agradecer una serie de sugerencias útiles de uno de los referees (como los paralelos de aibon o del grafito de Campello para eiar). No he incorporado otras, como la notable idea de que lo expuesto sobre śalbir encajaría con la teoría de la relación con silver cuya discusión precisaría de un amplio excursus. Hay que agradecer a ambos referees que llamaran la atención sobre el planteamiento demasiado optimista sobre la lectura śalitas. También he ampliado la explicación de algunos puntos, como kutur; pero consecuentemente, a fin de evitar una extensión excesiva, he abreviado o suprimido alguna otra no esencial. Originariamente explicaba que no entraba a valorar la cuestión del dual en edetano porque requeriría mucho
}

espacio y sería incorrecto despacharlo con una «opinión magistral». Pero visto que esta postura no ha sido comprendida, que no hay visos de que se publique una justificación en condiciones de ese "dual» (que era lo que yo esperaba), sino que, cual idolum theatri, se empieza a considerar dogma, y visto además que, aunque en 2008 ya presenté objeciones, quien lea Ferrer $(2010,70)$ o Ferrer et alii $(2015,14)$ podría pensar no solo que soy un entusiasta del dual edetano, sino que hasta entonces dudaba del «sistema dual»; deviene ineludible ser más explícito. Finalmente, quisiera remarcar que los cuadros acompañantes tienen mero sentido ilustrativo para facilitar la lectura, no argumentativos, pues no presentan datos homogéneos equivalentes. 
El objeto de este artículo es revisar una serie de fenómenos del consonantismo de la lengua íbera, especialmente pérdidas y mutaciones que no parezcan tener una causa morfológica. Aunque alguno de los casos puede encontrarse ya mencionado en la bibliografía, hasta el momento no se ha realizado ningún intento de sistematización ni de análisis, sino comentarios dispersos, siendo por ende difícil su localización ${ }^{1}$. Igualmente, aunque algún caso de los que expongo se menciona en mi índice crítico (Rodríguez Ramos 2014; en adelante NIC), por motivos de espacio y de tema, no hubo ocasión para su discusión específica.

Aun siendo obvio, conviene empezar explicitando que, por lo poco que se sabe de lengua íbera y lo exiguo de su documentación, no siempre puede darse una evaluación clara de los indicios, siendo algunos casos bastante probables o razonables, mas restando otros problemáticos. Ello no obstante, incluso estos merecen discusión.

En cuanto a los criterios de identificación de casos candidatos a ser considerados como posibles indicios de la presencia de los fenómenos, en general plantearemos dos tipos.

El primer tipo es el de la aparición de formas que parezcan ser variantes de otras conocidas. En estos casos hay que evaluar tanto la probabilidad de que se trate efectivamente de variantes del mismo término, como la posibilidad de una diferencia morfológica. Aun siendo difícil descartar de forma categórica las coincidencias, hay algunos criterios utilizables. Permitirán un análisis más seguro los términos más habituales y de contexto típico conocido; especialmente los formantes de compuestos, por ser un conjunto limitado de elementos isofuncionales y poderse cribar su variación por patrones de analogía. También es útil evaluar el grado de naturalidad fonética del cambio.

El segundo criterio de selección de elementos candidatos es el de las secuencias inhabituales. En especial los grupos vocálicos inusuales, que pueden hacer sospechar que haya habido una caída consonántica ${ }^{2}$, pero también la aparición de secuencias consonánticas infrecuentes resulta un indicio válido.

Resulta interesante observar que, tanto en los grupos vocálicos como en el resto de los fenómenos, acostumbra a jugar un rol el signo i. Este es un aspecto que convendrá revisar en el futuro, por si pudiera haberse visto favorecido por los criterios de análisis. Con todo, debe observarse que, efectivamente, la mayoría incluyen i y que entre los que no la incluyen no parece apreciarse una interpretación aparente. Por lo demás, siendo normal en las lenguas del mundo que una vocal /i/ afecte especialmente al consonantismo y conociéndose ya en íbero que i tiene una relación especial con 1 o s (Rodríguez Ramos 2000c, 268; 2004, 318-320), puede concluirse que no entra precisamente en la categoría de propuestas que precisen "pruebas extraordinarias».

\footnotetext{
1 Mención aparte merecen unos breves comentarios en Orduña $(2011 b, 132)$ sobre posibles casos de disimilaciones de algunos términos de la onomástica como bilos-iứ (iứ / biứ); giskeŕ / iskeŕ; untikesken / tuntike (pero el carácter onomástico del segundo es dudoso), a los que ańade como no disimilatoria la equiparación entre boto y oto. Como veremos en 2.7, prefiero no fiarme demasiado de los parecidos entre formantes. En todo caso sí trataré su interesante propuesta sobre la posible alternancia di-
}

similatoria del determinante ban con an. Orduña recalca acertadamente que "estas alternancias son particularmente frecuentes con la oclusiva labial», aunque para los casos que no serían disimilatorios no discute su posible motivación fonética.

2 Para un filólogo clásico puede ser ilustrativa la comparación con la etimología de términos griegos

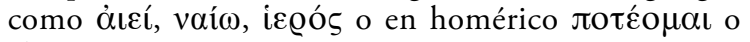
el genitivo -olo. 


\section{El CASO de los grupos vocálicos}

1.0. En íbero solo son frecuentes unos pocos grupos de dos vocales (en general aparentes diptongos decrecientes tipo ei, au, ai $)^{3}$, mientras que son especialmente atípicos los grupos vocálicos de tres miembros. Entre estos últimos existe una serie de ellos en los que la i ocuparía una posición medial, cabiendo sospechar que el grupo se haya producido por la caída de una consonante. De hecho, es probable que la /y/ consonántica no fuese un fonema realmente propio del íbero, sino secundario ${ }^{4}$. Esto no solo ha de ser una observación teórica, sino que se ha de ser consecuente: cuando parezca estar presente hay que buscar una explicación.

Dada la preferencia íbera por los «diptongos» decrecientes y dados los fenómenos de "sandhi» vocálico observados en los compuestos, cuando encontramos una secuencia de i más vocal puede sospecharse la existencia de un límite de morfema por medio o alternativamente que: o bien el mantenimiento de ambas "vocales» era necesario para mantener el sentido del término, o bien realmente ha caído una consonante que las separaba. Esto puede aplicarse a casos bien conocidos con ekiar, ekien, eŕiar y iunstir (vide 1.2) y, a su vez, a la posición proponible para una consonante presuntamente caída en los grupos trivocálicos con i medial: más probable tras la $\mathbf{i}$.

1.1. El primer caso a tratar es uno que hace tiempo que llamó la atención por su atípica estructura fonética, siendo el término más frecuente con grupo vocálico: el formante de compuestos aiun. La solución que se le ha dado es que se trataría de un préstamo exógeno en íbero (Correa 1994, 271), idea en la que ha influido, quizás demasiado, su parecido con un nombre celtibérico.

Naturalmente esta es una de las explicaciones posibles cuando se encuentra una estructura atípica. Sin embargo, de acuerdo con lo que sabemos de íbero, en concreto la escasa frecuencia del segmento $\mathbf{b u}^{5}$, cabe otra posibilidad: la de que provenga de una forma previa proto-íbera *aibun. De hecho, según lo que comentaremos, podría tenerse en cuenta la alternativa *ainun (cfr. $\$ 4.2$ ) o *aigun (cfr. $\$ 5$ y 1.4 ), por más que la propuesta más evidente es la primera ${ }^{6}$. La forma reconstruida sería comparable con la aparente marca de propiedad de la inscripción meridional (H.2.1, aibon-a / aibon-r), por más que la lectura alternativa aikon no es descartable.

1.2. Un mismo tipo de solución puede proponerse para el conocido término iunstir (y variantes), en el que tal vez tengamos un substantivo verbal formado con un prefijo $i$ - procedente de $e$ (Rodríguez Ramos 2000a, 30) y una raíz -u(n)st- o mejor -u(n)s-t- ${ }^{7}$. De nuevo, el timbre vocálico apunta a las tres soluciones: la evidente ${ }^{*} b u(n) s t$ - o las alternativas ${ }^{*} n u(n) s t-\mathrm{y}^{*} g u(n) s t$-; pero no puede descartarse una raíz con inicio vocálico. Hay que tener en cuenta que las variaciones de este

3 Sobre los grupos vocálicos puede verse el capítulo 7 "Secuencias vocálicas en interior de morfema» de Quintanilla (1998, 134-146).

4 Correa $(1994,4.14)$ se hace eco de su rareza indicando que el fonema parece existir, pero que su uso es restringido, seńalando su posible presencia en iunstir y clara en préstamos; mientras que en Correa $(2005,40)$ limita la presencia tanto de /y/ como de /w/ a préstamos.

5 Cfr. $M L H 1.1 \$ 412$ y 506.

6 Estando el estudio de estas caídas en situación incipiente, se hace difícil establecer un orden de pre- ferencia de más a menos probable. En principio, por su especificidad vocálica consideraría más probable una caída de $b$ - o de $n$-; considerando provisionalmente preferible la primera por ser un aspecto mejor establecido.

7 Como variantes tenemos iustir (E.4.2, F.9.6, La Joncosa), iuśtir (F.17.2, F.9.2), iumstir (D.0.1), iumstír (F.13.5), iunśtir (G.15.1), iunsir (F.9.7, H.0.1) y unsir (F.13.28). El que iunsir coincida con iunstir sugiere que sea una variante morfológica; mientras que es interesante observar que las formas con śs suelen ser en escritura meridional. 
término, con o sin $\mathbf{n}$ o $\mathbf{t}$, podrían ser indicio de una metátesis: lo que en su caso daría una cierta verosimilitud a una caída de una $n$ y quizás una raíz ${ }^{*} n u s(t)$-.

1.3. Otro caso conocido es el segmento baies (Silgo, Tolosa 2000) al que ya sus editores comparan con baites (cuya dental se documenta en dual catalán y grecoibérico como sonora d baides ${ }^{8}$ ). La segmentación de baies y su identificación con baites parecen razonables, habida cuenta: la frecuencia del término, el que suele acompañar a aparentes antropónimos y la propia rareza del grupo vocálico. En contra podría argüirse que el texto es singularmente breve para ser un documento con baites, que la forma de disco es atípica de los mismos y, en conjunto, que resulta demasiado agramatical en comparación. Es evidente que o se presuponía un contexto conocido o se complementaba la información con la casi ilegible cara B. En todo caso, la objeción no parece decisiva.

Puede considerarse que la posición de la consonante caída es la esperable, el paralelo aceptable y que la caída, especialmente de la sonora, en un entorno de palatalización no resulta extraña. Con todo, a falta de localizar más paralelos con pérdida de /d/, sería un fenómeno aislado.

1.4. Parece haber pasado desapercibida una serie de grupos vocálicos que suelen encontrarse al final de formas de aspecto verbal ${ }^{9}$ : biteian: bantúraś (H.0.1); śalir : biteian : bantứer[ (D.0.1); aŕikaler : eianiŕ (C.0.3); dedeiar ikane (B.7.34); teia : baneia (G.7.5; Fletcher, Bonet 1991$1992^{10}$ ). Casos a los que posiblemente haya que añadir el inicio del plomo C.0.3 (Velaza 1994) con la lectura salaiaŕkis-teian ${ }^{11}$ y tal vez el inicio del plomo C.1.5 lakeŕ-eiar y el final del grafito parietal C.18.2 -teiar.

8 En los casos en que lo considero conveniente y es razonable asumir que la inscripción corresponde al sistema dual, doy su transcripción como tal y la indico con el superíndice d. Aunque los trabajos de Ferrer han supuesto avances muy positivos (en especial la existencia de formas intermedias de algunos signos que unas veces son las formas marcadas y otras no) no considero todavía fiable el «dual» edetano. Es importante entender que no basta con decir que hay «dualidades» y transcribirlas adjudicándoles el valor fonético que nos parezca más natural. Para esto ya teníamos las transcripciones de Fletcher o el léxico de Silgo (1996). Es menester ver si hay coherencia, si hay uno o más sistemas en lo edetano y si es compatible con el dual catalán, pues mezclar sistemas diferentes contaminaría los datos. Pero mientras que para el sistema dual catalán tenemos el detallado análisis de Correa (1992), que es una demostración en toda regla, y también una comprobación de su coherencia según criterios de fonética sintáctica en mi tesis de 1996 (Rodríguez Ramos 2004, 243-255; 2005, 87 y 90) donde se da un fundamento interno a su valor fonético real, para el edetano la argumentación se reduce a unas líneas de una nota donde Ferrer $(2005$, 970, nota 65 ) indica que es aceptable porque la coherencia es del $80 \%$ frente al 90 del catalán. Dejando de lado problemas estadísticos como el mucho menor tamaño de la muestra (problema clave del edetano) o de evaluación (podría decirse que la incoherencia edetana es el 200\%), lo normal sería presentar una estadística verificable, no un «abstract». No suple esta deficiencia el que en sucesivos artículos se nos presente el tema como resuelto. Suponiendo inminente tal ampliación ya presenté (2008, 586 , nota 10 y 2014,96$)$ unos breves comentarios críticos donde se apuntaban algunas soluciones para su aprovechamiento. Así F.9.3 es interpretable como usando un dual invertido, con 8 casos de 8 determinables (y el que Ferrer no lo detectara ni como problemático incrementó mi escepticismo sobre su estadística); mientras que llamaba la atención sobre las oscilaciones paralelas, tras 1, de F.21.1, para las que podría sospecharse algún fenómeno fonético. El tipo de problemas que no se han resuelto es fácil de comprobar viendo que el propio dbaides aparecería consistentemente con te sorda en edetano (F.6.1 x2; F.9.3; F.9.8; frente a un único de en F.6.1). Cabe observar que De $\mathrm{Hoz}(2011,187)$ acepta las conclusiones de Correa (1992), pero matiza mucho más su extensión a lo edetano.

9 De hecho, creo que la totalidad de los términos aquí mencionados están analizados como probables términos verbales en la tesis de Orduña. Respecto a eianiŕ, puede verse cómo los análisis de Orduña (2005a, 195 y 206) concluyen que el sufijo -iŕ sería típicamente verbal.

10 Sobre las lecturas en íbero meridional, véase Rodríguez Ramos (2002b).

${ }^{11}$ Lectura sin correcciones innecesarias seguida en una ocasión por Untermann (1995, 247). 
Es interesante la comparación de los primeros con otras formas como ekian (La Camareta; Pérez Rojas 1993) o bitekian (B.3.2), por no hablar de los diversos ekiar de las marcas de autoría. De hecho, podríamos tener una típica marca de autoría con eiar en vez de ekiar en la inscripción meridional de Campello (Olcina 2001) que, con reconstrucción hipotética de la zona dańada, podría ser bi[lo]s-te-eiar-kuti, con kuti como posible complemento directo.

\begin{tabular}{|c|}
\hline H.0.1 iltukebeleś-e : núltun-ike : śanteten : bit-eian : bantứaś : \\
\hline D.0.1. ikośar-ka : *[]siko : en : śalir : bit-eian : bantứer[ \\
\hline C.0.3 śalaiaŕkis-t-eian / \\
\hline C. 0.3 śalaiaŕkis-te : beŕkebeś-a : aŕikaler : eian-iŕ \\
\hline B.7.34 d abelgiŕdi-ka : n-ted-eiạr-ikane : \\
\hline G.7.5 biśkibitérseti : teia : baneia : basbituŕbartin \\
\hline C.1.5 lakeŕ-eiar \\
\hline 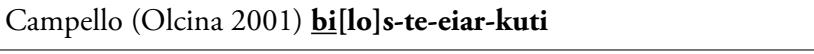 \\
\hline Gruissan eleŕ : kaisbor-a : bitekian \\
\hline Camareta kobeśir : ekian \\
\hline Frecuencia de fórmulas con ekiar equivalente a «fecit» \\
\hline
\end{tabular}

TABla I. Grupos-eia-

Si comparamos el fragmento : śalir : biteian con śalir : biteŕokan : (B.7.38) y ban : kutur : biteŕokan (B.7.38) se ve claro que, en los ejemplos que consideramos, -i- sería el núcleo verbal. Esta «raíz» ya por su tamaño debiera hacernos pensar en un verbo fundamental del léxico íbero, tal vez incluso un auxiliar; mientras que por su complemento (śalir), uno que admita un uso "transitivo». El cotejo con el bitekian de Gruissan, donde habría una raíz -ki- coincidente con la de ekiar, cuyo sentido equivalente a fecit, es básico, transitivo y adecuado para verbos auxiliares, encaja con el extrańo esquema trivocálico y la facilidad fonética para la caída de /g/ ante /i/, permitiendo plantear que, en estas formas -eia-, tengamos en realidad un -egia- subyacente (concordante con lo que apuntan las inscripciones en sistema dual).

Este tipo de fenómeno es normal. Un buen paralelo lo tenemos en el galo, donde hay alternancias con o sin «g» medial. Así Lambert $(2003,46)$ muestra varios ejemplos: la tribu de los Boii con una etimología en *bogii; la del antropónimo Maupennos desde *Magupennos; o variantes de antropónimos con y sin /g/ (Maiorix / Magiorix y Catuenus / Catugenus). Si lo unimos a la ausencia de $/ \mathrm{p} /$ en ambas lenguas, podría sospecharse un fenómeno de área lingüística.

\section{LA POSIBLE CAÍDA DE b- INICIAL}

Hay dos términos que han sido objeto de discusión específica al respecto. El primero es el inicio de aparente grupo verbal bite- (v.gr. biteŕoke), comparado con otros casos en que tendríamos 
ite-. El segundo es el de la ceca bolśken y su versión más moderna olśken de lo que acabará siendo «Osca». Para ambos casos, De Hoz (2001, 346; 2011, 282 y 343) ha propuesto un análisis morfológico como prefijo.

Merece observarse que las alternancias bo / o parecen fácilmente relacionables con la rareza del signo bu que apunta a una caída de $\mathbf{b}$ ante $\mathbf{u} ; \mathrm{y}$ que, aunque esta explicación no parece extrapolable a los casos de bi- (al menos directamente), llama la atención que hay diversos casos que coinciden en apuntar a fenómenos de caída de $\mathbf{b}$ ante $\mathbf{i}$ - $(\$ \$ 2.1,2.3,2.4,2.5$ y 2.6).

\begin{tabular}{|l|l|l|}
\hline bolśken / bon & olśken / on & $\begin{array}{l}\text { Uolciani / } \\
\text { «oscenses regionis uescitaniae» }\end{array}$ \\
\hline Neronken & & Narbo \\
\hline bieikate & iekate & \\
\hline & kaŕesoŕ : ieike & \\
\hline Inicio «verbal» bit-e & Inicio «verbal» it- & \\
\hline Formante bilos (muy frecuente) & ]ikárilos-ku & \\
\hline śalir muy frecuente en levantino & $\begin{array}{l}\text { Meridional: } \\
\text { * ausencia de salir } \\
\text { * śalbi- es frecuente }\end{array}$ & \\
\hline Rareza de grupo -lb- en levantino & & \\
\hline ausencia de śalbi- en levantino & & \\
\hline
\end{tabular}

TABLA 2. Alternancias $b / \emptyset$ más destacadas.

2.1. El caso de bite- merece ser tenido en cuenta para su cotejo con ulterior evidencia, pero en la actualidad no parece utilizable. No es imposible que exista un fenómeno fonético y, de hecho, hay algún paralelo indiciario a favor, pero, dada la variabilidad típica de los verbos, hoy por hoy la explicación morfológica parece preferible. En todo caso, mi impresión es que, más que un prefijo b-, lo que aquí tendríamos sería una alternancia entre bi- e i-.

2.2. Para el segundo, hay que decir de entrada que difícilmente puede proponerse el mismo prefijo b- que en el anterior, por ser el primero una forma verbal y este un topónimo. Por otra parte, es muy dudoso que se trate de una variación morfológica, pues en tal caso se esperaría encontrar paralelos de la misma en otros topónimos.

En cambio, la explicación fonética presenta diversas ventajas. Primero, que la excepcionalidad de la sílaba bu en íbero permite suponer una caída de la consonante $(/ \mathrm{bu} />/ \mathrm{u} /)$ y que el paralelo con la ceca neronken como forma íbera formada sobre el topónimo Narbo ya induce a pensar que el mismo fenómeno se estaba extendiendo desde la vocal más posterior a la siguiente. Encaja además con indicios de una pronunciación no plenamente oclusiva de la labial (tipo $/ \mathrm{v} / \mathrm{o} / \mathrm{w} /$ ) que permitirían explicar la base fonética del fenómeno (Rodríguez Ramos 2005, 24) ${ }^{12}$. Segundo, que

12 El tema mereció un breve comentario en Quintanilla (1998, 270s), aunque alguno de sus ejemplos presenta problemáticas especiales. 
en las monedas con bolśken aparece la marca bon, mientras que con las de olśken la marca on y que estas marcas aparecen en las cecas de la región. Tercero, nos encajaría la mención de Tito Livio $(21,19,8$ y 11$)$ a unos «volciani» con los que contactó Aníbal al cruzar el Ebro ${ }^{13}$. El fenómeno parece, pues, fonético y diacrónico.

2.3. Más difícil es el análisis de la comparación de bieikate (F.20.1) con iekate (La Joncosa; Ferrer 2006) y kaŕesoŕ : ieike (B.1.373; Solier \& Barbouteau 1988, 73-85), pues no parece haber datos analíticos que hagan probable su identidad (que -ke y -kate parezcan sufijos de caso limita el segmento a comparar), ni se ve clara qué función gramatical atribuirle (¿demostrativo?) ${ }^{14}$. Por otra parte, incluso aunque considerásemos que son términos de uso equivalente, si la forma es, como da la impresión, algún tipo de breve pronombre (biei- / iei-), no resultaría extraño encontrar dos pronombres diferentes de aspecto similar. Así las cosas, aunque es un paralelo apropiado para este tipo de alternancia bi- / i-, debe ser manejado con reservas.

2.4. Respecto a la cuestión de una alternancia entre \#bi- e \#i- fonéticamente motivada, hay información interesante en el íbero meridional; tanto en sus inscripciones como en la relación entre su sistema de escritura y el levantino.

En lo que concierne a la relación entre ambos sistemas de escrituras, hace tiempo que se ha observado la identidad formal entre el signo meridional bi y la u levantina ${ }^{15}$. Esta problemática en concreto, así como otras sobre la curiosa relación entre los silabogramas en i meridionales y sus derivados formales levantinos, las he tratado anteriormente (Rodríguez Ramos 2004, 86 y 88s). Lo interesante son los indicios de una pronunciación próxima a la u levantina, quizás de contextos de neutralización de la oposición entre ambos en archifonema.

La cuestión es que la forma de $\mathbf{u}$ levantina es precisamente la del signo bi meridional. Siguiendo paralelos en la historia de la escritura, puede interpretarse, y parece que ha de interpretarse, como que existió una pronunciación de la forma del signo cuyo inicio se confundía con la de la vocal $\mathbf{u}^{16}$. Una explicación fonética obvia sería la de una asimilación progresiva del rasgo de labialidad sobre la vocal con subsiguiente caída de la labial, similar a lo observado sobre bu; aunque los detalles pueden cambiar y puede haber tenido algo que ver la mencionada posible pronunciación semiconsonántica o aproximante-fricativa de $\mathbf{b}$ tipo /w/ de la que existen indicios en meridional ${ }^{17}$. Paralela y apuntando a

13 La relación entre «volciani» y Osca ya fue defendida por Tovar, aunque recibió un primer estudio en Pita (1952, 304, 305 y 306s), quien deja claro que en la época se consideraba un fenómeno diacrónico. Pita propone relacionarlo con el «oscenses regionis uescitaniae» de Plinio $(3,4)$, lo que proporcionaría otro elemento concordante.

${ }^{14}$ En el segundo y tercer casos parecerían estar determinando a un substantivo. Para el primero es interesante recordar la posibilidad de que bi sea un término numeral (dentro de la reconstrucción de Orduña 2005 b y 2011a) y cotejarlo con el probable numeral oŕkeiabaŕ-iekite (F.13.4) y el plausible abaŕ-ieikite (F.6.1). Claro que un numeral también es susceptible de recibir un determinante.

15 En cambio, la u meridional es el antecedente del ḿ levantino.
16 Este tipo de evolución se documenta en las runas en las que, al cambiar la pronunciación del nombre de algunas letras, estas adoptaron el valor de la nueva pronunciación. Así la runa «j» de nombre «jāra» pasa a ser «a» cuando por caída de la inicial su nombre pasa a pronunciarse «ár»; mientras que el antiguo signo para «a» ha cogido el del alófono posicionalmente motivado para a nasal al pasar de pronunciarse "ansuR» a "ãsur» (Spurkland 2005, 81s). El paralelo es especialmente curioso en tanto que una posibilidad similar para explicar el paso de la u meridional a notar una vocal nasalizada en levantino sería perfectamente plausible.

17 Existen indicios de que en meridional (o en alguno de sus dialectos) la vocal /i/ era especialmente inestable, con tendencia a asimilar rasgos de su entorno. De esa manera, la forma meridional ku es la que es interpretada (u «oída») como ki en levantino, mientras que a su vez la 
esta última opción es la evolución del be meridional hacia el signo e levantino, desechando la forma e meridional, por más que en este caso parece más bien tratarse de un desdoblamiento, puesto que el be levantino formalmente parece proceder de una variante cursiva del meridional.

Si examinamos el testimonio interno, propio del meridional, hay dos cuestiones relacionables con una aparente caída de $\mathbf{b}$ ante $\mathbf{i}$, aunque las dos son de índole muy distinta. Ambas las trataremos en los dos apartados siguientes.

2.5. En la inscripción de Baeza (Correa 1989) se encuentra un segmento ]ikárilos-ku. Es plausible identificar un sufijo -ku con aparente valor de marca de agente en meridional ${ }^{18}$, lo que sugiere que lo que le precede sea un antropónimo. La lectura de los tres primeros signos no es todo lo segura que cupiera desear ${ }^{19}$, pero parece razonable y podría especularse con una variante del formante de compuestos ekaŕ (NIC n. $\left.{ }^{\circ} 55\right)$. El ilos restante recuerda al formante bilos (NIC n. $\left.{ }^{\circ} 45\right)^{20}$, tanto porque este es frecuentísimo como, sobre todo, porque en íbero los finales en -os no lo son. Como hemos visto casos de aparente caída de $\mathbf{b}$ inicial y en concreto alternancias $\mathbf{b i} / \mathbf{i}$, nada podría encajar mejor que una alternancia bilos / ilos. ¿Problemas? Varios.

En primer lugar, la interpretación de ikaŕilos como compuesto antroponímico es plausible, pero recae demasiado en identificar un sufijo -ku, en la alta frecuencia de bilos y el que no parece haber alternativas para comparar con ilos. En segundo, un problema concreto: lo que le sigue es bekibilos, con un claro aspecto de compuesto de tipo onomástico, precisamente por bilos (vide $N I C$ n.o 44/2). Realmente no es del todo imposible que una misma forma aparezca con dos pronunciaciones diferentes en una misma inscripción, sea por motivos de fonética sintáctica o porque sean nombres de personas de zonas con dialectos diferentes (p. ej. Hernández y Fernández). Puede recordarse incluso que hay inscripciones que tienen grafías contradictorias de un mismo término o criterios de segmentación de palabra diferentes (cfr. F.6.1). Aun así, este ejemplo, aunque aducible por la falta de explicaciones alternativas a ilos, resulta discutible.

2.6. Otra posibilidad digna de tener en cuenta, porque podría aproximarnos tanto a cuestiones dialectales como al proto-íbero, pero que en la actualidad es muy problemática, es el paralelo entre una forma que presenta bi en meridional con formas con $\mathbf{i}$ en levantino. El caso es que en íbero levantino y en grecoibérico conocemos bien el término śalir, que es frecuente en documentos sobre plomo y cuyo sentido de valor monetario es ampliamente conocido. Aunque el número de plomos en meridional es reducido, sorprende la ausencia de śalir en los mismos.

Hasta el momento su único posible paralelo detectado, pero nada claro, es el segmento ]śelir de G.7.2, de un texto tan fragmentado que ni aporta datos contextuales útiles, ni permite saber siquiera si el término está completo.

ki meridional podría ser el origen del ku levantino. De forma similar hay una discordancia sobre la forma ti meridional, que resulta siendo te en levantino. No cuesta mucho proponer una posible relación: $b$ es una labial y una /i/ labializada es una /ü/; $k$ es una velar, es decir, de punto de articulación posterior $\mathrm{y}$ una /i/ posteriorizada es una $/ \mathrm{u} /$. Menos natural sería la explicación para la dental, aunque podría especularse con una apertura de la vocal por un punto de articulación dento-alveolar de la $t$.

18 En meridional el «sufijo» -ku es utilizado en estructuras donde en levantino esperaríamos un -te marca de agente y en otras parece ser un alomorfo de -ka (Rodríguez Ramos 2002b, 238 y 2004, 265ss). Aunque en alguna inscripción la forma del signo permitiría especular con que en realidad se tratara de un signo ka marcado (¿con valor de -k ?), la forma con que aparece en otras inscripciones con el mismo uso parece descartar la idea.

${ }_{19}$ El primero está fragmentado y para los otros dos no sería imposible una lectura como tu y a.

20 Presentado como comparación segura por Orduña (2011b, 132). 
En cambio llama la atención que dentro del reducidísimo elenco de plomos meridionales sí se repita una forma śalbi- inédita en levantino: aitikeltun-ku : iunśtir : bekor : śalbitas : oteŕoketa (G.15.1); bikứtibaś-ku : śalbi[ (F.9.2); ] : katulu : alabultun : śalbiŕiar (o śalbibaiar) (El Amarejo; Broncano 1989). En concreto, la posición en G.15.1 recuerda la de algunos documentos con śalir como B.7.38. La confirmación podría estar en F.17.1 donde śalitas se compararía con śalbitas, pero actualmente la lectura es problemática ${ }^{21}$.

Es interesante recordar que esta interpretación concuerda con el carácter excepcional del grupo -lb- en íbero y podría indicar una evolución desde un «salbi-» o «salwi-» original con preservación del grupo en meridional, pero resta problemática.

2.7. Dejo para el final una serie de posibles comparaciones de formantes de compuesto con bfrente a otros sin ella. El motivo principal para su «marginación» es que, aunque en algún caso se intenta presentar este tipo de comparaciones entre formantes con o sin tal o cual consonante como autoevidentes (es decir, que su mero listado sería prueba suficiente), las considero documentación de cotejo; interesante como referencia, pero poco fiable sin una criba crítica.

Objetivamente hay que reconocer que alguno de los paralelos candidatos puede defenderse con una buena argumentación. Pero un caveat importante lo supone el que los formantes iniciados con b-son los más frecuentes. De ello se derivan dos cuestiones: que hay más posibilidades de que se produzca un parecido por coincidencia; y, sobre todo, que el número de posibles alternancias detectables es un porcentaje muy pequeño del total de formantes con $\mathbf{b}$ - inicial. Por ello difícilmente puede ser un fenómeno generalizado, siendo más probable que se trate de casos aislados con explicaciones específicas. Hechas estas advertencias, veamos los paralelos candidatos con su número del NIC:

\begin{tabular}{|c|c|}
\hline ban (n. $\left.{ }^{\circ} 25\right)$ & an $\left(\mathrm{n} .^{\circ} 8\right)$ \\
\hline beike (n. $\left.{ }^{\circ} 31\right)$ & ike / eike (n. $\left.{ }^{\circ} 63\right)$ \\
\hline bene (n..$^{37)}$ & ene (n. $\left.{ }^{\circ} 57\right)$ \\
\hline biứ (n.o 50) & iứr (n.o 72) \\
\hline boto (n. o 54$)$ & oto (n. o 110$)$ \\
\hline
\end{tabular}

De estos hay que observar que la existencia de un formante beike es dudosa, mientras que la de ene es probable, pero no del todo clara. Sin embargo, es relevante observar que estos 4-5 parecidos se producen sobre un total de 29 formantes indexados con b inicial. Aun reconociendo que, dado lo comentado de las alternancias bi / i, la comparación entre biứ e iuŕ resulta fascinante y es po-

21 La autopsia de Fletcher (un experto en examinar plomos y cuyo material gráfico es reproducido en los $M L H)$ indica claramente śalitas, pero Ferrer $(2005,967$, nota 51) indica que todos tienen el trazo horizontal adicional que los convertiría en bo. De hecho, en las fotos de Fletcher se aprecia el trazo adicional en un caso de la cara $A$, pero no en uno de la $B$ (el resto es poco visible), por lo que es posible que considerara adventicio el de A (que no dibuja), que es la impresión que da, pues parece seguir por el signo anterior. El problema se repite en el otro término con el signo, para el que Fletcher ve claro el cuarto trazo en el caso de A, pero no en los dos de B. 
sible que en el futuro haya que volver a ella ${ }^{22}$, considero que solo merecen una presentación detallada dos de ellas: an y oto.

La primera es compleja porque ban no es precisamente frecuente y el que de los tres casos posibles el único claro provenga de una inscripción celtibérica induce a suspicacia. Por otra parte, es sabido que ban tiene al menos otros dos usos homófonos y seguramente más. De an, en cambio, hay al menos dos casos que parecen claros, aunque no sean muy típicos.

En todo caso, traigo este par a colación en tanto que sobre sus homófonos ha presentado Orduña (2011b, 133-136) una idea muy interesante. Orduña no analiza sendos términos por sus apariciones en los compuestos onomásticos sino dentro de un estudio sobre afijos en el que plantea posibles disimilaciones (en contextos en que en primera instancia los interpretaría como determinantes). Sugiere que el elemento ban pueda aparecer como an en algunos casos en los que la b habría caído por disimilación de la $\mathbf{b}$ siguiente. Hay algunos considerandos y matizaciones interesantes que se podrían hacer a esta propuesta de la que no puedo asegurar que sea correcta, aunque no es descabellada, pero no considero que sea esta la ocasión para tal digresión. Sí que procede mencionar que, si bien esa disimilación podría aplicarse al compuesto an-bels $(N I C 8 / 1)$, no se puede en an-talskar $(N I C$ 8/5). Sí que es cierto que darle un cierto valor determinante a an- delante al menos alguno de los compuestos de tipo onomástico ${ }^{23}$ sería una solución digna de consideración en vista del carácter más "clítico" que de formante que indico en $N I C$ n. ${ }^{\circ} 8$, nota (lo que tendría el mérito objetivo de resolver una irregularidad). Lo que no queda claro es si se pueden forzar todos los casos «onomásticos» a determinados por (b)an-, siendo especialmente problemático antalskar. Existe además el problema de peso de que, de ser así, esperaríamos una clara muestra concordante de «onomásticos» determinados por ban.

Respecto al par boto/oto indico en NIC una interpretación plenamente ortodoxa que podría explicarlo como una alternancia. Según lo comentado en 2.2 sobre la aparente pérdida de $\mathbf{b}$ ante $\mathbf{u}$ y los indicios de progresiva extensión del fenómeno a la otra vocal posterior $(\mathbf{o})$, puede considerarse verosímil una caída de la $\mathbf{b}$ de boto y, al no ser un fenómeno regular sino en progresión, encajaría con la coexistencia de ambas variantes. Debe tenerse en cuenta, no obstante, que hay problemas respecto a si la forma es con «t» o con «d» (puesto que sus ejemplos más claros serían BODONILUR y BILOS-OTON), lo que haría dudosa su equiparación, pero que los datos no son incontrovertibles ${ }^{24}$, por lo que habrá que esperar a tener más documentación.

\section{Apariciones «COMPENSATORIAS» DE $\mathbf{i}$}

La evidencia de esto es muy difícil de establecer y la misma clasificación como compensatorias, es decir, que se produce una i para compensar la pérdida de otro fonema, no es más que una con-

22 Cabe observar que el grupo vocálico permite plantear una caída de consonante, siendo una peculiaridad que los asemeja más. Las formas originarias más plausibles serían **bibuŕ, **biguŕ o incluso **binuŕ (véase 4.2). Para la primera, aparte de la caída de $\mathbf{b}$ ante u podría plantearse una disimilación que diera origen a las dos formas base: ${ }^{* *}$ bibuŕ > biuŕ / ${ }^{* *}$ ibuŕ $>$ iứ. Pero naturalmente esto es solo una muestra de las posibilidades del método de análisis; en principio, no veo evidencia que haga probable que biuŕ e iúr sean lo mismo.

23 Esta interpretación como determinante delante de onomástico está en cierta manera implícita en lo que indica Orduña, aunque no llega a dejarla clara y plantea expresamente la posibilidad de que sea el formante de compuesto ban.

24 Me remito a lo comentado en el NIC en sus respectivas entradas, especialmente a no 110/3. 
jetura, pudiendo entenderse tanto como que la i representa directamente la mutación de una consonante, como que supone el alargamiento con fractura de la vocal previa.

3.1. En primer lugar, destaco una posibilidad que relaciona a dos demónimos bien conocidos, laietanos y lacetanos. Su comparación es interesante en tanto que se ha discutido mucho la relación entre ambos, su parentesco o incluso su posible identidad; por más que a la postre la considero escasamente utilizable. En los compuestos íberos se conoce bien laké́ / lake- pero no laie-, que sí documentamos como demónimo en la ceca laie-sken.

En laiesken y «laietani» no queda claro si hemos de considerar que el nombre en sí es lai- (que sería lo más simple) o laie- con «sandhi» (como p. ej. untikesken). Una forma lai resulta un tanto atípica, mientras que laie presenta un grupo vocálico sospechoso con $\mathbf{i}$ en posición medial consonántica. Dada esta anomalía, resulta tentador sospechar que en laie haya habido una alteración consonántica y, dada la relación entre ambos términos, es tentador suponer que la forma base sea precisamente el término lake.

En el lado positivo hay que señalar que este tipo de fenómeno no es nada extraordinario y es fácil encontrar paralelos, como en castellano desde el latín «ley» (lat. leg-em) o en inglés desde el germánico "day» (cfr. al. Tag). Con todo, estos fenómenos suelen relacionarse con procesos de palatalización que acaban produciendo el diptongo, en cuyo caso se esperaría poder encontrar más indicios en íbero. A falta de más paralelos analógicos, resulta una interpretación problemática.

De hecho, conforme a lo que hemos venido comentando, pueden plantearse alternativas en las que la forma fuese *laiCe o incluso *laCie, perspectiva desde la cual podría proponerse una relación con latie (NIC n. ${ }^{\circ}$ ) $)$, puesto que, independientemente de si es de origen galo o no, parece probable que tuviese un uso en la antroponimia íbera. Es la estrecha relación entre los lacetani y los laietani lo que hace más probable el primer enfoque.

3.2. El fenómeno de yotización de consonante ya fue propuesto por Orduña (2005a, 58; 2008, 287) para la reconstrucción de un posible paradigma de flexión nominal de términos con el sufijo -ar. El término principal es la comparación entre uśtalar y uśtalaibi, para el que propone que el grupo ai provenga del ar ante el sufijo bi. La idea es sugestiva y meritoria, pero presenta algunos problemas, por lo que puede considerarse una hipótesis de trabajo pendiente de comprobación ${ }^{25}$.

Con todo, parece oportuno comparar el ejemplo de Orduña de uśtalar (de uśtalarilune F.9.5) frente a uśtalaibi (F.13.2) con otros ejemplos de sufijo -bi; puesto que nos muestran una realidad más compleja. Así ya en Rodríguez Ramos $(2004,269)$ mostraba los casos de baites-bi, bati-bi y aŕikaŕ-bi. Lista a la que puede añadirse el doble topónimo śaitabi / śaitir, hasta ahora sin explicación. Tendríamos, pues:

25 La idea de Orduña se complementa con la sugerencia de entender el CASTLOSAIC (H.6.1) como un ergativo ibérico donde -AIC provendría de los sufijos ar y ka. Sigue en esto, como casi todo el mundo, la idea de Hübner $(1893,178)$ de que el término "parece» significar castulonense y la interpretación de Untermann $(1990 / 2,653)$ de que -SAIC sería un grupo de sufijos ibéricos. La interpretación como agente es plausible, en tanto que, como indica Untermann, no es seguro que sea una inscripción funeraria; pero no es realmente seguro que se trate de origo, cabiendo otras alternativas, como que se tratara de un cargo (tipo "pretor de Cástulo»). Más crítico es el que no está tan claro que la inscripción esté en íbero, pues hay indicios como mínimo de dialectalización (cfr. Rodríguez Ramos 2010, 131). Como uno de estos indicios son precisamente los grupos vocálicos, esto podría ser suficiente explicación para SAIC. 


\begin{tabular}{|l|l|l|l|}
\hline batir & batibi & & \\
\hline baites-ir & baites-bi & baites & \\
\hline aŕikal-er & ariká́-bi & aŕikaŕ & \\
\hline śaitir & śaitabi & & \\
\hline uśtalar & uśtalaibi & & cfr. ustar-ike (F.20.1) \\
\hline
\end{tabular}

Hay que observar algunas cuestiones previas.

Primera, que dada la excepcionalidad de los finales en $\mathbf{l}$ y la oscilación que se observa entre $\mathbf{l}$ y las vibrantes, no es extraño que una forma aŕikal-o uśtal- en final absoluto adopte un final en vibrante, como ocurre con aŕikaŕ, y como pudiera pasar en ustarike.

Segunda, que tanto las formas de baites como las de batir parecen calificar a personas. Para la primera he propuesto un significado similar a «testigo» o "garante», para la segunda ya De Hoz propuso que fuese una magistratura por su repetición en el plomo de Palamós (C.4.1), un poco al estilo de los bintis del primer bronce celtibérico de Botorrita. Su aparición conjunta parece indicar que batir sea jerárquicamente superior a baites en los documentos, lo que encajaría con un oficial, magistrado-notario. Sobre sus variantes, baitesir, baitesbi y batibi los plomos permiten diversas interpretaciones, desde tipos de determinación a sufijos sintácticos. Como para baites y batir parece probable un valor de substantivo, este parece presumible para aŕikal- ${ }^{26}$.

Tercera, que, si añadimos el par śaitir / śaitabi, al proceder ambos de epígrafes numismáticos y con la confirmación del SAETABIS latinizado, hemos de suponer que ambas formas tienen un valor de substantivo, lo que hace difícil entender -bi como sufijo sintáctico.

Naturalmente no puede descartarse por completo que sea una coincidencia, pero la interpretación de śaitabi como forma sufijada paralela a las otras resolvería algunas cuestiones. El topónimo śaitir sería comparable con baites-ir y aŕikal-er, de lo que puede suponerse una forma originaria del tipo śait- o śait-V. De śait $(V)$ + -ir tendríamos śaitir (con o sin «sandhi» vocálico); mientras que de śait $(V)$ + bi tendríamos śaitabi donde la segunda a o bien recogería el timbre originario (*śaita) o sería una vocal epentética o similar.

Es interesante observar que esta interpretación sería compatible con la sugerencia de Ferrer (2009, 454 nota 9) hecha extrapolando lo planteado por Orduña, de que en batibi, baidesbi, uśtalaibi y aúikaŕbin tuviéramos un determinante numeral bi(n) «dos». En cierta manera le supone un apoyo, en tanto que relacionarlo con śaitabi hace difícil darle un sentido de caso sintáctico. Pero aun así, esta explicación deja puntos obscuros. Si las formas con -bi son «dos», ¿qué sentido tienen las formas -ir / -er? ¿Realmente esperamos que intervengan tantos personajes por parejas, pero no en otras cantidades? Porque no tenemos los hipotéticos *baites-iŕu, *baites-laur o similares ${ }^{27}$.

26 De hecho, hay indicios que apuntarían a que aŕikal- refiriera a una función «legal» (más que a un cargo), pero son poco concluyentes.

27 Orduña $(2005,403$ y 2011, 132) apunta a un numeral ilun «3» con ejemplo en uśtalar-ilune. Esto no afecta aquí, en tanto que no hay indicios de que fuese un magistrado o una persona. En todo caso, si la comparación es con baites y aŕikal-, se preferiría ** uśtalilun-. Obsérvese, para la segmentación uśtal-ar tanto que como ejemplifican los formantes de CTO es excepcional que un substantivo íbero acabe en vocal -a, como el paralelo ustar-ike (F.20.1). 
En todo caso, la cuestión con estos paralelos es que cuando se les añade -bi no crean nunca diptongos y que, de hecho, las alternancias baites / baitesbi / baitesir y aŕikaŕ / aŕikaŕbi / aŕikaler indicarían que la forma -ar de uśtalar sería substituida por -bi y no la precedería, por lo que, según ello, esperaríamos un *uśtal-bi / *uśtaŕ-bi.

Naturalmente es demasiado pronto para extraer conclusiones definitivas y podría intentar proponerse que las formas batir y śaitir fueran formas base con la -r incluida y que igualmente la forma base no fuese *uśtal sino uśtalar y que en las otras no apareciera diptongo por ser «temas» en -i; pero creo que no hace falta seńalar lo artificioso que resulta sin apoyos adicionales. En cualquier caso, los paralelos actuales van en contra de la idea de que la -ai de uśtalai-bi sea un resultado de un ar originario ${ }^{28}$.

\section{LOS INICIOS SINGULARES DE $\mathbf{l}$ Y $\mathbf{n}$}

Esta es una cuestión sobre la que ya en su momento hice algunas observaciones dentro de estudio comparado de formantes de compuestos onomásticos y de un léxico experimental de segmentos no onomásticos (Rodríguez Ramos 2000c, 268). En él llamaba la atención sobre la ausencia de inicios en li- y nu-.

4.1. Respecto a li-, este sigue siendo un inicio extrañísimo. Es significativo que cuando se encuentra suele tratarse de antropónimos extranjeros. Así tenemos los tres casos de likine, dos musivarios (E.7.1 y K.28.1) y uno sobre cerámica campaniense (Bonet \& Mata 1989, Valencia II) y el galo lituŕíś (B.7.34). En las rupestres de Osséja (Campmajó \& Ferrer 2010) se ha podido identificar una secuencia de dos términos likuŕ-talaŕi, que sigue siendo tan excepcional que no altera la conclusión ${ }^{29}$.

A priori hay dos explicaciones estándar para esta ausencia. O bien se ha producido una palatalización de la 1 - inicial y ha pasado a ser una especie de /y/ desapareciendo su notación gráfica $\left({ }^{*} \#\right.$ li > \#i); o bien se trataba de una «l» velar (como en ruso o en catalán) que ha alterado el timbre vocálico de cualquier /i/ originaria. Da ambas, parece preferible la primera porque: el carácter velar de la lateral no es evidente y no se han identificado indicios concordantes ${ }^{30}$; el que el factor palatalizante de /i/ es universal y parece adaptarse mejor a que el fenómeno se dé en inicios absolutos

\footnotetext{
28 Por otra parte, la extraordinaria frecuencia de finales en -r (para el que he propuesto que sirve de indicio para sospechar que el término es un substantivo) y las grandes limitaciones de consonantes íberas posibles en posición implosiva y de final absoluto, plantean la posibilidad de que esta -r sea un archifonema resultante de la neutralización de diversas consonantes en posición final. Desde este punto de vista sería normal que ante sufijos hubiese un resultado diferente según la consonante originaria. Con todo, la opción de que -(V)r sea un sufijo derivativo nominalizador es perfectamente viable, por más que, de hecho, el suponer la existencia de tal sufijo no excluye la posibilidad de que parte de la frecuencia se relacione con fenómenos de neutralización consonántica.

29 Dejando aparte posibles variaciones dialectales (los términos de las inscripciones de la Cerdaña
}

son en ocasiones extraños), una hipótesis es que sea un término exógeno (cfr. Rodríguez Ramos 2014, 87 , nota 10). Una interpretación como «ligur» es seguramente optimista en exceso, pero no descartable; mientras que el semánticamente interesante liquor no parece tener un uso epigráfico que lo haga verosímil. Como antropónimo, Ligur es obviamente conocido, mientras que Talaris / Talarius existe (cfr. Solin 2009, 173s), pero es tan excepcional y problemático que resulta poco más que una curiosidad. Por otra parte, en una inscripción del s. II de Roma se atestigua un cognomen Thalarus (AE 1993, 305) de probable origen griego pero que, con el permiso de teleuś, tampoco parece una solución evidente.

30 Lo comentado en 4.3 no es precisamente un apoyo. 
(donde es natural un refuerzo de tensión) pero menos en posición medial ${ }^{31}$; y la consideración de que, si se tratase de una lateral velar que afectara al grupo li-, extraña la ausencia de efecto en términos como iltiŕ o iltur ${ }^{32}$.

4.2. Por su parte, nu- no solo está ausente en inicio absoluto, sino que es infrecuente incluso en medial. De los casos en que se localiza la secuencia en posición medial, en varios es manifiesta o probable la existencia de un límite de morfema entre $\mathbf{n}$ y $\mathbf{u}$ (iakikin-ứr-e, ḿŕríkin-ứr-e, anbośiltun-u, baiseltun-u y posiblemente ilurargirenug; aunque de suspecta); siendo llamativo el que solo aprecio un caso al norte del Ebro. De hecho, la secuencia nu tiene menos frecuencia que bu, cuya rareza en íbero es conocida desde hace tiempo (Untermann 1990.1, $\$ 412$ y $\$ 506$ ). Es legítimo tomar en consideración que en posición medial la «mayor» presencia de bu tiene mucho que ver con la variante del sur de Francia (que usa bứ como variante del formante boŕ o presenta el nombre galo kanbulo), pero debe recordarse que de bu tenemos hasta algún ejemplo en posición inicial y no del sur de Francia (buranalir, buistiner).

Aunque es menester ponderar el hecho de que en íbero los inicios en $\mathbf{n}$ - son relativamente poco frecuentes y que, además, las primeras sílabas con vocales posteriores son también las menos frecuentes, la ausencia resulta sospechosa.

Una primera explicación sería suponer una labialización de $/ \mathrm{n} /$ hacia $/ \mathrm{m} /$ (alófono de $/ \mathrm{b} /$ en íbero) y consecuentemente asimilaríamos el caso a bu, lo que presenta obvias ventajas explicativas. Otra sería relacionarlo con la cuestión de las nasalizaciones vocálicas progresivas en íbero (Rodríguez Ramos 2000b; 2004, 307-314) y suponer una eventual neutralización del timbre vocálico. Esta neutralización podría suponer, p. ej., que alguno de los casos en que encontramos notación na- (que es relativamente frecuente) procediera de otro timbre vocálico originario. Considero preferible la primera explicación.

Aparte de estos dos casos más llamativos, existen algunas cuestiones sobre inicios en $\mathbf{l}$ y $\mathbf{n}$ merecedores de atención.

4.3. Así, en el mismo estudio mostraba la ausencia de inicios en lo- entre los formantes de compuesto, por más que sí se localizaban en el léxico de comparación. La cuestión es que, aunque hay que ser prudente ante la escasez de datos de contraste, existen algunas consideraciones obvias sobre los pocos casos de inicios en lo- documentados ${ }^{33}$.

El primer ejemplo es el único caso donde no hay un paralelo que lo ponga en duda y donde, aunque no sea seguro, es probable que sí tengamos un inicio en lo-. No se trata de un inicio absoluto, pero sí que parece que habría un límite de palabra ante él y que no sería una secuencia de sufijos. Es el caso de ]ltibeleś-te-lokiŕ de F.20.3, por más que no sería descartable que fuese un «verbal» elokiŕ, que no deja de recordar las formas en ŕok, quizá con disimilación regresiva por el sufijo (¿¿*e-ŕok-iŕr??).

El segundo caso sería el final dokárkige-etan del plomo B.1.373. Su primer elemento recuerda mucho al elemento lekaŕ identificable en diversas inscripciones ${ }^{34}$. Como la alternancia e/

31 La secuencia li en medial se encuentra en términos bien conocidos como śalir, kalir o tolir-bitaner, habiendo varios ejemplos en términos menos frecuentes.

32 Obsérvese que la $\mathbf{l}$ íbera parece tener una especial relación con e. Así, siendo rarísima la aparición de -1 en final absoluta, sí tenemos los formantes tetel y sekel.
33 Obsérvese que en posición medial la secuencia lo no solo está bien documentada, sino que es sospechosa su frecuencia como inicio de segunda sílaba de formante de compuesto onomástico (aloŕ, beloŕ, bilos, tolor) o variantes (kolo < koŕo). Con todo, en varios casos podría plantearse una disimilación de «rhótica» (p. ej. *aŕo-ŕ > alo-ŕ).

34 B.7.35, B.7.36, F.20.3, el plomo de Ruscino (Rebé et alii 2012) y quizás F.13.3. 
o en íbero, sin ser extraordinariamente frecuente, está bien documentada (cfr. Quintanilla 1998, 11.3.1), al ser un paralelo mayoritario puede sospecharse que en lokaŕ tengamos una variación sobre lo que propiamente sería lekaŕ.

El tercer caso es el término loŕsa(r), para el que resulta interesante comparar sus dos ocurrencias en plomo con un tercero:

\section{B.7.36 \\ C.2.3}

\author{
${ }^{\mathrm{d}}$ basiŕiute : loŕs / ar : tứrśildiŕ : \\ d : taśkaliŕs / loŕsa : batibi :
}

\section{B.1.373 ${ }^{\mathrm{d}}$ utarige : ursa : ebośirige}

Aunque la evidencia es muy débil, si unimos el paralelo con ursa, la aparente explicación de lokaŕ como variante de leká́ y la escasa frecuencia de inicios en lo-, hay base para plantearse la hipótesis de una caída de /l/ ante /o/. Si tenemos en cuenta que los inicios en lu- también son poco frecuentes, podríamos sospechar una relación con el timbre posterior de la vocal; pero ya hemos indicado que los timbres de sílaba inicial en /u/ son poco frecuentes y prácticamente inexistentes los inicios en li-.

4.4. Volviendo a los inicios absolutos en $\mathbf{n}$ - hay una cuestión curiosa. Resulta que en estos es mucho más frecuente que a la vocal le siga una consonante no oclusiva, o incluso otra vocal, que el que le siga una consonante oclusiva $\left(\mathbf{b}, \mathbf{k} / \mathbf{g}\right.$ o t/g $\mathbf{g}^{35}$; que representaremos con el símbolo convencional C).

Así, de inicios en $\mathbf{n}$ con esquema nVC- no tenemos ningún caso con los timbres e, o y $\mathbf{u}$, mientras que con a solo tenemos nabaŕsosin que, por lo demás, es una lectura discutida ${ }^{36}$.

Ante este panorama parece definitivamente significativo que encontremos casos de ni- y que lo haga en "palabras" que presentan un aspecto similar y en general complejo: niteketane y nitebetane junto a banitekitane (La Joncosa; Ferrer 2006); ${ }^{\mathrm{d}}$ nigokadiáru y ${ }^{\mathrm{d}}$ nigogaiadai (plomo de Ampurias), nikin[ y nite (plomo de Monteró 2; Ferrer et alii 2009); y el nikiteikul[ de un grafito de Manises (Lobregat \& Rosser 1993) de autenticidad dudada ${ }^{37}$.

Aunque no comparto los calcos vascoibéricos de prefijos verbales que al estilo Silgo hace Ferrer $(2006,135$ s, 143 y 151) al comentar la inscripción de La Joncosa, la excepcionalidad de la estructura nVC- que presentan estos inicios en ni- plantea como explicación obvia el que sea un morfema, por más que seguir el sendero del parecido con prefijos vascos parece como mínimo prematuro $^{38}$.

\footnotetext{
35 Difiero aquí la cuestión de las aparentes pronunciaciones no completamente oclusivas de $\mathbf{b}$.

36 Prefiero la lectura de su editio princeps, pero ante la extrańa forma del tercer signo, Untermann, en los $M L H$, propuso la corrección nalbesosin que no es completamente descartable.

37 No encuentro justificada la lectura nikiteiskul[ (Velaza 2001, 661) a partir de uno de los diversos «resbalones» del escriba, cuya impericia y peculiar trazado de ku apuntan a la falsedad de la pieza.
}

\footnotetext{
38 Los planteamientos de comparación directa sin criba crítica debieran evitarse, porque en el mejor de los casos son «apuestas» (del tipo a ver si al final resulta que sí) y, en el peor, argumentaciones circulares. Una afirmación vale lo que su argumentación. P. ej. cuando Ferrer compara la posición de kứs con la mí para acabar equiparándolo con el vasco "gu» "nosotros», no solo es dudosa la equiparación posicional, sino que se olvida una criba tan obvia como ¿̨realmente esperamos que los objetos se refieran a sí mismos en plural mayestático?
} 
Frente a esta excepcionalidad de formas nVC- se observa que el número de casos en que el tercer elemento no es una oclusiva es bastante respetable, con la mencionada excepción de la falta de inicios en nu. Podemos citar la palabra nai y los formantes de compuestos neitin, neŕse, nios y niś; así como los casos nauiba, nauŕa, nauki[, naltun[, naltinge, nanban, neitailiniŕe, neia[, dneitegeŕu, nisoŕba-, noukoś, norotaŕ y el dudoso naŕtokui[ sospechoso de ser un antropónimo exógeno.

Merece observarse el gran porcentaje de grupos vocálicos, en especial ei y au, tras los cuales sí que puede aparecer una oclusiva. Es como si estructuralmente el aparente diptongo funcionara como una sílaba cerrada. Realmente el fenómeno es sorprendente, pero de difícil explicación ${ }^{39}$.

Un cotejo con el otro fonema sonante similar que puede aparecer en inicial absoluta, la 1-, no parece de gran ayuda, puesto que su distribución es solo parcialmente concordante. En estructura IVC- solo es excepcional cuando la vocal es i, o o u; pero ya hemos visto que lo que sucede es que son infrecuentes en cualquier inicio absoluto. En lo referente a las otras dos vocales (a y e) ambos contextos se aprecian bien con la-, mientras que con e el testimonio es inverso: bueno respecto a leC-, pero cuando no le sigue una oclusiva lo normal es que estemos con formas en lei- / leis- del formante NIC n. ${ }^{\circ}$ 97. Posiblemente aquí tenemos algún fenómeno fonético subyacente que produce la repartición entre los timbres de a y de e. ¿Tal vez una apertura de timbre ante sonante haya convertido varias /e/ originarias en $/ a /$ ? ¿O es una posteriorización? Aunque ambas ideas son concordantes con la ausencia de inicios en li-, la cuestión queda abierta. En todo caso no parece que puedan englobarse las peculiaridades de $\mathbf{n}$ y de $\mathbf{l}$ en un modelo común.

Resulta evidente que, para poder dar una explicación clara al problema suscitado por los inicios en $\mathbf{n}$-, convendría poder determinar si la escasa frecuencia de inicios en $\mathbf{n}$ - en íbero estaba ya presente en proto-íbero o no. Si estaba, si originariamente ya era escasa, entonces la frecuencia de formas diptongadas es excepcional y habría que buscar un origen a su formación. Por el contrario, si no estaba (es decir, si n- en proto-íbero no era un inicio infrecuente), entonces las formas diptongadas serían interpretables como un resto cuantitativamente normal, preservadas por el contexto.

Si hubiese concordancia con la lateral, lo que yo propondría es que la aparente diptongación ante oclusivas se debiera a una semiconsonantización de una consonante implosiva, de forma similar a lo que he llamado « $i$ compensatoria». Una explicación tal, encajaría con el modelo de escasez originaria de $n$-. Pero, en ausencia de concordancia, la hipótesis más sencilla sería suponer que en los casos de nVC-, siendo C una oclusiva, se habría producido una nasalización de la vocal y una coarticulación. Tiene la ventaja adicional de concordar con el fenómeno de la nasalización vocálica ya ha sido descrito en íbero (Rodríguez Ramos 2000b). En concreto respecto a ḿ, donde variantes como nabaŕ / ḿbaŕ / UMAR y quizás incluso abaŕ mostrarían un proceso de desaparición de la notación $\mathrm{nV}$ ante consonante.

Desde esta perspectiva sería la presencia de una semivocal en diptongo, o de una consonante implosiva (es decir, posiblemente ser sílaba larga o cerrada) la que habría impedido la nasalización y permitido la conservación de la n. Este modelo sería compatible con una pérdida de nasales iniciales proto-íberas. La solución tendrá que esperar a que se conozcan mejor tanto los "diptongos» íberos, como el papel de /n/.

39 Pero obsérvese que concuerda con lo indicado en Rodríguez Ramos $(2004,317)$ sobre el papel silábico de la semiconsonante /y/. 


\begin{tabular}{|l|l|l|l|}
\hline Inicios nVY- nVR- ¿sílaba larga? & & Inicios NVC- & \\
\hline nai & palabra & nabaŕsosin & C.1.6 \\
\hline nauiba & (Bonet \& Mata 1989) & nikin[ & (Ferrer et alii 2009) \\
\hline nauki[ & F.9.9 & dnigogaiadai & C.1.24 \\
\hline naứa & F.20.3 & dnigokadiarú & C.1.24 \\
\hline neia[ & H.0.1 & nite & (Ferrer et alii 2009) ¿morfema ni-? \\
\hline neitaliníre & F.9.5 & niteketane & (Ferrer et alii 2009) imorfema ni-? \\
\hline dneitegeŕu & C.2.3 & nitebetane & (Ferrer et alii 2009) ¿morfema ni-? \\
\hline neitin & palabra, formante CTO & nikiteikul[ & (Lobregat \& Rosser 1993) suspecta \\
\hline nios & formante CTO & & \\
\hline noukoś & B.5.1 & & \\
\hline naltinge & G.1.1 & & \\
\hline naltun[ & (Ferrer et alii 2009) & & \\
\hline nanban & G.7.2 & & \\
\hline Neŕse & Formante CTO & & \\
\hline niś & Formante CTO & & \\
\hline nisoŕba & B.7.35 & & \\
\hline norotá́ & B.7.34 & & \\
\hline naŕtokui[ ? & B.7.38 & & \\
\hline NALBE- ? & Formante CTO & & \\
\hline
\end{tabular}

TABLA 3. Comparaciones de $n V$ - seguido de vocal o consonante no oclusiva frente los de $n V$ - seguidos de consonante oclusiva.

\section{Posible CAÍdA DE VELAR INICIAL}

El caso conocido es el de utur (D.0.1; Untermann 1989) para el que se suele aceptar la muy verosímil idea de Untermann de que es una variante del conocido kutur. Para esta caída consonántica hay, en principio, dos explicaciones. La primera es que es que cayera por aspiración ${ }^{40}$, lo que concordaría con un fonema sordo $/ \mathrm{k} /$. La segunda es por un tipo de coarticulación por similitud fonética, lo que concordaría con una sonora /g/. Por desgracia, la documentación disponible no

40 Como aspiración lo interpreta Orduña (2011a, 130 nota 11). Esta propuesta está motivada por los problemas de interpretar abaŕ como «diez» equivalente al vasco «hamar». Aunque la cuestión es interesante (aun siendo improbable, no puede descartarse por completo la existencia de aspiraciones en íbero estándar), es un problema secundario. Bastaría con suponer que en protoíbero se hubiese perdido la aspiración de un hipotético proto-íbero-vasco, sí conservada en vasco. El fenómeno es trivial y hay muchos paralelos. 
permite determinar la sonoridad de la consonante inicial, aunque los casos más claros apuntan a una sorda ${ }^{41}$.

De ser sorda, la explicación más evidente sería una caída por aspiración. Esto encajaría con las propuestas de fonética diacrónica vasca desde Martinet (donde las oclusivas tensas originarias se habrían perdido por aspiración), pero en íbero tenemos un buen repertorio de "tensas» iniciales. Tampoco se documenta la aspiración en los antropónimos indígenas en escritura latina y griega en la zona propiamente íbera. Tampoco es favorable el testimonio de las inscripciones íberas donde debieran identificarse más alternancias con o sin k- inicial. Sí que se podría plantear la posibilidad de que fuese un cambio activo $/ \mathrm{k} />/ \mathrm{kh} />/ \mathrm{h} /$ y que, de hecho, bajo la grafía de $\mathbf{k}$ - tuviésemos $\operatorname{aspiradas}^{42}$, pero ni acaba de explicar la ausencia de alternancias, ni, sobre todo, las grafías latinas y griegas. La única posibilidad que se me ocurre es que fuese un cambio incipiente que empezara afectando a las vocales posteriores, para lo que existe algún paralelo ${ }^{43}$. Ello justificaría la falta de otros testimonios, pero resulta un tanto forzado.

Más normal sería el cambio en caso de ser $/ \mathrm{g} /$, pues en los sistemas de oclusivas distinguidas por rasgo de sonoridad, tras la /p/ (ya inexistente en íbero), el siguiente fonema más proclive a caer es la /g/. Además, una /u/ puede causar una labialización; y una /g/labializada sería próxima a /w/ y a /b/ para cuya caída ante /u/ hay claros indicios en íbero. Este fenómeno tendría la ventaja de ser congruente con lo indicado en 1.4 , pero naturalmente habrá que esperar a tener más datos.

\section{Otros FENÓMENOS CON LATERALES Y VIBRANTES}

No entraré a repetir en este apartado lo que ya he expuesto en otros lugares sobre las alternancias $\mathbf{l} / \mathbf{r} / \mathbf{r}^{44}$. Dado lo natural del fenómeno fonético, considero que los ejemplos aportados son suficientes $y$, aunque hay alguna cuestión que podría añadirse ${ }^{45}$, no altera las conclusiones.

6.1. El primer caso que examinaré es interesante en tanto que, siendo fonéticamente verosímil, permitiría aclarar la documentación de un extraño y excepcional sufijo. Este se encuentra en

41 El grecoibérico apuntaría a «g», pero no parece posible que tras gud[ (G.4.1) siga una u, mientras que gudu (G.1.2) podría ser guru y proviene de una inscripción de autenticidad discutible. En dual catalán la distinción de ku es problemática, en especial por depender de un mero punto en inscripciones a menudo en mal estado. Ferrer (2014) lee dkutu en B.7.38 y B.23.3. La primera es de un plomo mal conservado. La segunda, que en principio es el más claro ejemplo, de una inscripción rupestre de Osséja de edición antigua y por desgracia no es raro que surjan cambios notables en las autopsias en este tipo de inscripciones, por lo que me cuesta confiar en ella para un detalle crítico. Finalmente, dentro de la idea de Ferrer de que el término proveniente de los signarios, en los duales (que se supone serían precisamente los primigenios) la secuencia es ${ }^{\mathbf{d}}$ gutu.

42 De forma similar en castellano medieval algunas grafías con "f-» son en realidad grafías por /h/ (cfr. Ariza $1990,102 s s)$.
43 En la familia de lenguas mongolas es habitual la aspiración de $/ \mathrm{k} / \mathrm{y}$ que este fenómeno comience por cuando está ante vocal posterior (Rybatzki 2003, 373).

44 Rodríguez Ramos 2002a, 29s; 2003, 343 y 348; 2005, 32 A.2.1. Un breve párrafo al respecto también en Quintanilla (1998, 254).

45 En todo caso resulta «fascinante» descubrir alguna de estas cuestiones inéditas publicadas en primicia por Silgo $(2011,318)$ a partir de una comunicación personal mía a tercera persona. Licencia curiosa por parte de quien en otras ocasiones sin venir a cuento decide glosar ciertas críticas falaces contra mí y entonces decide que lo que al respecto si he publicado replicándolas no merece ni referirse, ni por alusiones. Aunque las publicaciones de Silgo suelen seguir criterios más que discutibles, hay cosas que simplemente son inapropiadas. 
los términos uŕkekeŕeŕe (F.6.1) y aŕeŕe (F.9.7) ${ }^{46}$. El análisis por defecto del primero es el de un compuesto de tipo onomástico uŕke-keŕe seguido de un enigmático sufijo -ŕe; siendo la alternativa postular un poco probable error del grabador, quien habría repetido los dos últimos signos ${ }^{47}$. El análisis del segundo no es tan evidente, pero parece verosímil relacionarlo, como hacía Untermann $(1990,1 \$ 556 \mathrm{~s})$, con la serie de términos que clasificaba de modo aproximativo como «paradigma pronominal», siendo posiblemente el que más aspecto de pronombre real tenga. Más en concreto con aŕe-ka (F.20.1), aŕe-n (H.9.1), además del conocido aŕe de las estelas y posiblemente aŕeta (B.7.37). Este aŕe- podría ser similar al más frecuente en iŕ-, con el que es posible que guarde relación.

Difícilmente puede ser casualidad el que en ambos casos la sílaba previa sea también -ŕe- y sería poco sistemático ignorar un hecho tan evidente. Mi propuesta de explicación es la siguiente: en ambos casos lo que tendríamos es el conocido sufijo -te en forma de /-de/ y se habría producido una asimilación progresiva de la dental. Se trataría, pues, de un alomorfo fonéticamente condicionado y sendos términos serían equivalentes respectivamente a *úrkekeŕe-te y a *aŕe-te. Parece relevante el que ambos casos provengan de yacimientos próximos de la provincia de Castellón, hecho coherente con una interpretación como fenómeno morfo-fonético dialectal.

Es obvio que esta asimilación progresiva sería más factible si el sufijo fuese con la sonora /-de/. Por desgracia, el carácter sordo o sonoro del sufijo no puede determinarse de forma categórica, puesto que el testimonio al respecto de las inscripciones con marca dual no es claro (en lo que tal vez tengan que ver cuestiones de «sandhi» y pronunciaciones con caída vocálica). Lo que sí es cierto es que, alomórfica o forma base, la versión con notación sonora existe.

6.2. Otro caso interesante es el comentado en NIC n. 95 sobre el probable antropónimo de aspecto galo dkatulatie- y el compuesto de aspecto más íbero dultiladie, respecto a su posible comparación con la «firma» baise-tatie.

Los dos primeros términos pueden analizarse tanto como nombres cien por cien íberos, como alternativamente como cien por cien celtas, pero, como la hipótesis celta requiere de alguna licencia para ello, la interpretación íbera resulta preferible. En todo caso no es descartable que se tratase de un término celta incorporado al repertorio onomástico íbero. El tercero, por su apariencia de «firma» permitiría segmentar un formante onomástico tatie para el que no se conocen paralelos. Pero aquí podría plantearse que la consonante inicial sea una sonora /d/ y, dado que las alternancias $\mathrm{d} / \mathrm{l}$ no son extrañas y que incluso podrían ser indicio de la adaptación de un término exógeno, tatie podría ser variante de latie.

6.3. Otro ejemplo llamativo, aunque con una problemática curiosamente similar al ilos / bilos de la inscripción de Baeza, es el del misterioso formante de compuestos onomásticos bios. Aunque es un término al que se le ha prestado excesiva atención, hasta hace poco era un absoluto «hápax».

Con todo, poco altera este estatus la aparición de dos probables segmentos con aspecto de compuesto de tipo onomástico setibios y baŕkabios en un plomo de origen irregular (Velaza 2004). De un lado, porque presenta aspectos sospechosos que sugieren que sea una falsificación. De otro,

\footnotetext{
46 Untermann $(1990,1, \$ 539)$ incluye en esta serie etaŕe, pero parece preferible la segmentación etaŕ-e.

47 Orduña $(2005 a, 321)$ considera que hay un sufijo e con una ́r antihiática, argumentando que es improbable que exista un sufijo -ŕe. Posiblemente,
}

como al tratar de -ŕok- (Orduña 2005a, 122), se basa en la ausencia de inicios en ŕr- en íbero; pero es sabido que las limitaciones de inicio de palabra no tienen que coincidir necesariamente con las de raíz o morfema. 
porque al ser dos testimonios de algo excepcional en un mismo documento, tanto puede entenderse como indicio de falsificación, como de un fenómeno local dialectal. En el mismo sentido ahondan singularidades como uŕtieiuŕ en el mismo plomo, o boioi en su acompañante. Testimonio problemático en el mejor de los casos.

La cuestión es que al tratarse el biosildun del plomo grecoibérico G.1.3 de un testimonio tan singular, cabe darle una explicación singular. Puede considerarse, y tiene cierta lógica, que se trate de un error del autor por bi<l>osildun. Esto puede apoyarse en que el anterior es un bilosg[e]ŕe con un bilos correcto. Aunque sorprende un poco el que en un plomo tan pequeño se deje un error tan evidente sin corregir, es perfectamente posible.

La otra solución es la que ya apunté en $N I C$ n. ${ }^{\circ}$ 48: la de que tengamos el resultado de una palatalización de la lateral con una evolución tipo bilos $>$ billos / biyos $>$ bios. Esta propuesta tiene a su favor a nivel genérico el que es una evolución lingüísticamente típica; mientras que a nivel específico el de la no presencia de inicios en li- en íbero, fenómeno de tendencia concordante que apunta a la existencia de pérdidas de «l» por palatalización regresiva ante /i/. Lo que sí tiene la propuesta en contra, es el que en el mismo plomo aparezca un bilos. No sería imposible proponer una explicación plausible (como que biosildun proviene de una zona en cuyo dialecto se ha producido la palatalización, mientras que bilosgeŕe de otra), pero es un contra-argumento de peso y por ende la propuesta debe dejarse como hipótesis de trabajo pendiente de comprobación.

6.4. Una cuarta cuestión que quisiera comentar se deriva de lo observado al investigar las secuencias de fonemas íberos. En Rodríguez Ramos (2003, 344) señalaba que, aunque por la excepcionalidad de los grupos - $\mathbf{l k}$ - y su paralelismo con la de los grupos -lb-, podría pensarse en una evolución de la lateral a vibrante en dichos contextos (es decir ${ }^{*} \mathbf{l} \mathbf{b}>\mathbf{r} \mathbf{b}$ y ${ }^{*} \mathbf{l k}>\mathbf{r} \mathbf{k}$ ), de hecho, la secuencia estaba documentada en un término extraordinariamente frecuente: el formante balke (NIC n. $\left.{ }^{\circ} 24\right)$. Aunque existe algún testimonio de la probable variante baŕka- que nos encajaría con la mencionada tendencia fonética, es tan marginal que sigue echándose en falta una explicación a dicha singularidad.

Con los conocimientos actuales no parece poder identificarse una causa fonética que la justifique. Como mucho, tal y como sugería respecto a nalbe (NIC n. $\left.{ }^{\circ} 100\right)$, podría apuntarse a un mantenimiento por entorno nasal. Pero esto es poco probable, puesto que habría que justificar la presencia de un alófono nasal de $\mathbf{b}(/ \mathrm{m} /)$.

A falta de justificación contextual, la explicación estándar alternativa sería suponer que el mantenimiento del grupo consonántico se debiera a tratarse de un préstamo de otra lengua. El préstamo es el origen habitual de grupos consonánticos inéditos o "perdidos» en las lenguas; como ejemplifica en castellano el grupo -fg- en «afgano». Intuitivamente creo que es probable que la solución correcta vaya justo en este sentido, pero objetivamente cabe advertir de que las posibilidades más evidentes presentan dificultades tal vez insalvables.

Así, en el diccionario de galo de Delamarre $(2001,56)$ puede encontrarse el término balco «fuerte» con un uso en la construcción de antropónimos celtas. El sentido sería perfecto, pero, de hecho, si balco es un buen ejemplo de algo, lo es de las deficiencias de Delamarre en el uso de datos procedentes de la península ibérica. Aunque existe el adjetivo común en irlandés, ninguno de los antropónimos aducidos por Delamarre puede considerarse válido (de forma escasamente sorprendente son íberos) y en otros estudios sobre galo no he encontrado ni la forma. Las posibilidades de que se incorporara a la onomástica íbera un término que, de existir en céltico de la zona, no tenía uso antroponímico, son prácticamente nulas. 
El otro ejemplo es mucho más interesante. Es bien conocida la raíz semita malk «rey». Tendríamos, pues, un término usado en onomástica, con un sentido fundamental y esperable en la onomástica íbera precisamente con un uso frecuente, el tipo de término que podría haber pasado fácilmente de la lengua fenicia al íbero y, además, con una nasal inicial susceptible de favorecer el mantenimiento del grupo -lk-.

Sin embargo, tanto en fenicio como en púnico, el vocalismo de la raíz semítica ha pasado a /i/, siendo «milk». No descarto por completo que pueda llegar a explicarse un cambio de vocalismo tal en íbero, pero no parece sencillo. Por otra parte, dado que en la época de formación de la cultura íbera el arameo tuvo un enorme uso como «lingua franca», quizás pueda tenerse en cuenta la posibilidad de una presencia en occidente o incluso participación de población aramaico-parlante en la colonización, puesto que el arameo conserva la forma malk $^{48}$; pero es obvio que con los datos actuales no sería más que una especulación de aspecto improbable.

Mi conclusión es que, aunque la idea de que balke provenga de un préstamo del semítico malk «rey» sería una explicación interesante, en la actualidad ha de dejarse como mera posibilidad a tener en cuenta, pues presenta problemas de difícil solución que, más allá de las apreciaciones subjetivas, objetivamente la descartan.

\section{EPÍLOGO}

En este artículo se ha procedido a examinar una serie de indicios de cambios fonéticos en íbero basados principalmente en alternancias y en grupos inusuales de fonemas (como li-, lo-, nu-, -nu-, bu-, - $\mathbf{1 k}$ - o los grupos de tres vocales). Estos fenómenos permiten una aproximación a la evolución diacrónica del íbero y del proto-íbero.

Se aprecian irregularidades en los grupos vocálicos, principalmente trivocálicos con i medial. De un lado, algunos términos en eia posiblemente sean alternancias de ekia- con valor verbal. De otro, el examen de los segmentos infrecuentes en íbero apunta a que esta sea la explicación de aiun que tal vez provenga de *aibun. En el mismo sentido se ha planteado que, dada la estructura silábica íbera, cuando nos encontremos con un grupo de $i$ más vocal puede sospecharse un límite de morfema o incluso la caída de una consonante.

También se aprecian diversos casos de caída de b-. Varios de estos siguen la pauta esperable de ser ante vocal posterior, pero también hay indicios de caída ante $\mathbf{i}$ que en ocasiones parecen relacionables con una asimilación de los rasgos de la $\mathbf{b}$ sobre la vocal.

Otras cuestiones son más específicas, pudiéndose resaltar: la explicación del sufijo -ŕe como una asimilación progresiva de ámbito dialectal; tatie (entendido como /datie/) como variante de latie; la posibilidad de que bios muestre simplemente una palatalización de bilos; o los indicios de que el formante balke sea un préstamo exógeno, tal vez de origen semita. También puede tener interés resaltar la propuesta de relacionar las variantes toponímicas śaitir / śaitabi con paralelos como baites-ir / baites-bi y lo que ello podría aportar para explicar la alternancia i/a.

48 En arameo y en formas hebreas sufijadas (donde no hay epéntesis ni segolización) se mantiene la forma malk frente al innovador milk del fenicio (Gzella 2011, 451). Los testimonios de nombres fenicios en asirio, griego y latín de la pronunciación fenicia son abundantes y consistentes (Friedrich \& Röllig $1999 \$ 193$ a). Los casos de apertura vocálica hacia $e$ que se documentan (ibid. $\$ 91$ a) no parecen solventar el problema. 
Por otra parte, en el estudio se llama la atención sobre la extraña distribución de algunos inicios silábicos, especialmente los de en $\mathbf{n}$-, que varían aparentemente según siga sílaba cerrada o larga por naturaleza; siendo un claro indicio de un fenómeno diacrónico todavía no precisable.

\section{BiBLIOGRAFÍA}

Ariza Viguera, M., 1990, Manual de Fonología Histórica del Español, Madrid: Editorial Síntesis.

Bonet, H., \& C. MAtA, 1989, «Nuevos grafitos e inscripciones ibéricos valencianos», APL XIX, 131-148.

Broncano, S., 1989, El depósito votivo ibérico de El Amarejo, Bonete (Albacete), EAE 156, Madrid: Ministerio de Cultura.

Campmajo, P., \& J. Ferrer i Jané, 2010, «Le nouveau corpus d'inscriptions ibériques rupestres de la Cerdagne (1): premiers résultats», Palaeohispanica 10, 249-274.

Correa, J. A., 1989, «La inscripción vascular indígena hallada en Baeza (Jaén)», APL XIX, 183-189.

- 1994, "La lengua ibérica», Revista Española de Lingüistica 24-2, 263-287.

- 2004, "Elementos no indoeuropeos e indoeuropeos en la historia lingüística hispánica», en R. Cano (ed.), Historia de la lengua española, Barcelona: Ariel, 35-57.

Delamarre, X., 2001, Dictionnaire de la langue gauloise, Paris: Errance.

FERrer I JANÉ, J., 2005, «Novetats sobre el sistema dual de diferenciació gràfica de les oclusives sordes i sonores», Palaeohispanica 5, 957-982.

— 2006, «Nova lectura de la inscripció ibèrica de La Joncosa (Jorba, Barcelona)», Veleia 23, 129-170.

— 2009, «El sistema de numerales ibérico: avances en su conocimiento», Palaeohispanica 9, 451-479.

— 2010, «El sistema dual de l'escriptura ibèrica sudoriental», Veleia 27, 69-113.

— 2014, «Ibèric kutu i els abecedaris ibèrics», Veleia 31, 227-259.

FERrer i JANÉ, J., et alii, 2009, «Els materials arqueològics i epigràfics de Monteró (Camarasa, la Noguera, Lleida): troballes anteriors a les excavacions de l'any 2002», QPAC 27, 109-154.

Ferrer i JanÉ, J. et alii, 2015, «Towards a systematisation of Palaeohispanic scripts in Unicode: synthesising multiple transcription hypotheses into two consensus encodings», Palaeohispanica 15, 13-55.

Fletcher, D., \& H. Bonet, 1991-1992, «Bastida VI. Nuevo plomo escrito de La Bastida de les Alcuses (Mogente, Valencia)», Anales de Prehistoria y Arqueología. Universidad de Murcia 7-8, 143-150.

Friedrich, J., \& W. Röllig, 1999, Phönizisch-punische Grammatik. 3 Auflage, neu bearbeitet von Maria Giulia Amadasi Guzzo unter mitarbeit von Werner R. Mayer, Roma: Editrice Pontificio Istituto Biblico.

Gzella, H., 2011, "Northwest Semitic in General», en S. Weninger (ed.), The Semitic Languages. An International Handbook, «Handbücher zur Sprach- und Kommunikationswissenschaft» 36, Berlin/Boston: De Gruyter Mouton.

De Hoz Bravo, J., 2001, «Hacia una tipología del ibérico», en F. Villar, M. a P. Fernández (eds.), Religión, lengua y cultura prerromanas de Hispania, Salamanca: Universidad, 335-362.

- 2011, Historia lingüistica de la peninsula ibérica en la antigüedad: Vol. II. El mundo ibérico prerromano y la indoeuropeización, Madrid: CSIC.

Hübner, E., 1893, Monumenta Linguae Ibericae, Berlin: G. Reimer.

LAMbert, P.-Y., 2003, La langue gauloise, Paris: Errance.

Lobregat, M., \& P. Rosser, 1993, "Un fragmento cerámico con grafitos bilingües, y la pervivencia del sustrato ibérico en la ciudad romana del Tossal de Manises (Albufereta, Alicante)», LQNT 1, 119-135.

Olcina Domenech, M. H., 2001, "Grafit ibèric», Llegats del MARQ, Alicante: Museo Arqueológico Provincial de Alicante, 32.

Orduña Aznar, E., 2005a, Segmentación de textos ibéricos y distribución de los segmentos, tesis doctoral.

- 2005b, «Sobre algunos posibles numerales en textos ibéricos», Palaeohispanica 5, 491-506.

— 2008, "Ergatividad en ibérico», Emerita LXXVI/2, 275-302.

— 2011a, «Los numerales ibéricos y el protovasco», Veleia 28, 125-139. 
— 2011b, «Prefijos y clíticos en ibérico», ELEA 11, 131-151.

Pérez Rojas, M., 1993, «Las inscripciones con escritura tartésica de la cueva de La Camareta y su contexto onomástico. (Aportaciones sobre la "celtización" del mundo ibero-tartésico)», Antigüedad y Cristianismo $\mathrm{X}, 139-266$.

Pita Mercé, R., 1952, «Gentilidades y ciudades del pueblo ilergete», Argensola 12, 293-320.

Rébé, I., J. De Hoz \& E. Orduña, 2012, «Dos plomos ibéricos de Ruscino (Perpignan, P.-O.)», Palaeohispanica 12, 211-251.

Rodríguez Ramos, J., 2000a, "La lengua íbera: en busca del paradigma perdido», Revista Internacional d'Humanitats 3, 23-46.

- 2000b, "Vocales y consonantes nasales en la lengua íbera», Faventia 22/2, 25-37.

- 2000c, "Aproximación fonético-estadística a los compuestos nominales de la lengua íbera», QPAC 21, 259-270.

- 2002a, «Problemas y cuestiones metodológicas en la identificación de los compuestos de tipo onomástico de la lengua íbera», Arse 36, 15-50.

— 2002b, «La escritura ibérica meridional», Zephyrus 55, 231-245.

— 2003, «Sobre los fonemas vibrantes y afines de la lengua íbera», Veleia 20, 341-349.

- 2004, Análisis de Epigrafía Íbera, Anejos de Veleia. Series Minor 22, Vitoria-Gasteiz.

- 2005, "Introducció a l'estudi de les inscripcions ibèriques», Revista de la Fundació Privada Catalana per a l'Arqueologia Ibèrica 1, 13-144.

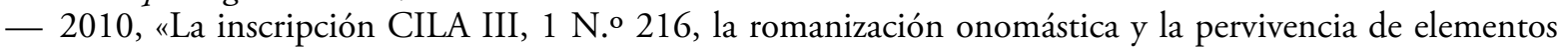
indígenas en la Cástulo Romana», Veleia 27, 123-134.

- 2014, «Nuevo Índice Crítico de formantes de compuestos de tipo onomástico íberos», Arqueoweb 15/1, 81-238.

Ryвatzki, V., 2003, «Intra-Mongolic taxonomy», en J. Janhunen (ed.), The Mongolic Languages, London: Routledge, 364-390.

Silgo Gauche, L., 1994, Léxico ibérico, Valencia: Real Academia de Cultura Valenciana.

— 2011, "Miscelánea ibérica y vasca», ELEA 11, 315-326.

Silgo, L., \& A. Tolosa, 2000, «Plomo ibérico escrito del Camp de Morvedre», Arse 34, 39-44.

Solier, Y., \& H. Barbouteau, 1988, «Découverte de nouveaux plombs, inscrits en ibère, dans la région de Narbonne», RAN 21, 61-94.

Solin, H., 2009, «Analecta Epigraphica CCLII-CCLVIII», Arctos 43, 161-190.

Spurkland, T., 2005, Norwegian Runes and Runic Inscriptions, Woodbridge: Boydell Press.

Untermann, J., 1989, «Nova inscripció ibèrica sobre plom, procedent del país dels Ilergetes», Acta Numismatica 19, 39-44.

- 1990, Monumenta Linguarum Ibericarum. Band III. Die iberischen Inschriften aus Spanien, Wiesbaden: Dr. Ludvig Reichert Verlag.

- 1995, «La lengua ibérica: nuestro conocimiento y tareas futuras», Veleia 12, 243-256.

VelazA, J., 1994, «Sobre dos plomos con escritura ibérica: una revisión y una noticia», Epigraphica 56, 9-28.

- 2001, "Chronica epigraphica Iberica II. Novedades y revisiones de epigrafía ibérica», en Villar, F., \& M. ${ }^{a}$ P. Fernández (eds), Religión, lengua y cultura prerromanas de Hispania, Salamanca: Universidad, 639-662.

— 2004, «Dos nuevos plomos ibéricos en una colección privada», Palaeohispanica 4, 251-261. 